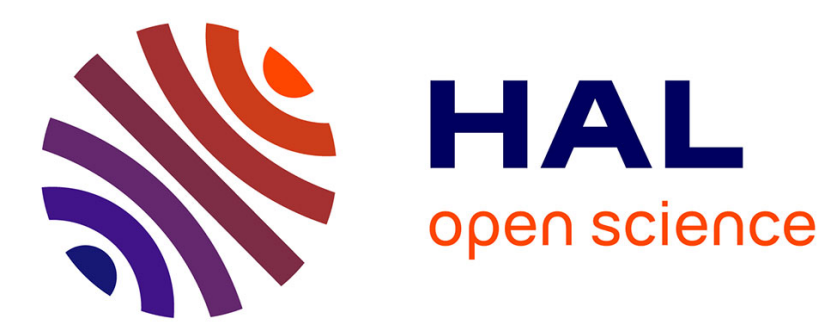

\title{
Statistical detection and isolation of additive faults in linear time-varying systems
}

Qinghua Zhang, Michèle Basseville

\section{To cite this version:}

Qinghua Zhang, Michèle Basseville. Statistical detection and isolation of additive faults in linear time-varying systems. Automatica, 2014, 50 (10), pp.2527-2538. 10.1016/j.automatica.2014.09.004 . hal-01081899

\section{HAL Id: hal-01081899 \\ https://inria.hal.science/hal-01081899}

Submitted on 2 Apr 2021

HAL is a multi-disciplinary open access archive for the deposit and dissemination of scientific research documents, whether they are published or not. The documents may come from teaching and research institutions in France or abroad, or from public or private research centers.
L'archive ouverte pluridisciplinaire HAL, est destinée au dépôt et à la diffusion de documents scientifiques de niveau recherche, publiés ou non, émanant des établissements d'enseignement et de recherche français ou étrangers, des laboratoires publics ou privés. 


\title{
Statistical detection and isolation of additive faults in linear time-varying systems *
}

\author{
Qinghua Zhang ${ }^{\mathrm{a}}$, Michèle Basseville ${ }^{\mathrm{b}}$ \\ ${ }^{a}$ INRIA Rennes Bretagne Atlantique, Campus de Beaulieu, 35042 Rennes Cedex, France \\ ${ }^{\mathrm{b}}$ IRISA, Campus de Beaulieu, 35042 Rennes Cedex, France
}

\begin{abstract}
This paper describes a statistical approach to fault detection and isolation for linear time-varying (LTV) systems subject to additive faults with time-varying profiles. The proposed approach combines a generalized likelihood ratio (GLR) test with a recursive filter that cancels out the dynamics of the monitored fault effects. To our knowledge, the proposed recursive filter is new for the considered faults. The resulting algorithm handles fault isolation with weaker assumptions than usual, in particular regarding the requirements on the number of sensors and on the stability of the monitored system. Numerical results for leakage detection in a gas transportation network illustrate the effectiveness of the proposed method.
\end{abstract}

Key words: Linear time-varying systems; additive faults with time-varying profiles; recursive filter; GLR test.

\section{Introduction}

The increasing requirements on higher performance, efficiency, reliability and safety for various industrial systems, together with the emergence of stronger safety and environmental norms in most of human activities, call for continuous research investigations in the field of fault detection and isolation (FDI). Since many industrial processes rely on physical principles, which write in terms of equations, providing us with mathematical models, it is reasonable to assume that a model of the monitored system is available; see e.g. [29] for a recent survey. Model-based approaches to FDI problems have been mostly studied for linear time invariant (LTI) systems; see for example [6, 12, 18, 21, 23, 30, 31, 40], and references therein. In many applications, however, this is too much a simplified assumption: quite often the timevarying and/or nonlinear properties of the monitored system cannot be neglected. Some more recent studies have been focused on nonlinear system FDI, such as those reported in $[5,8,17,20]$, but these results are often developed under restrictive assumptions, limiting their

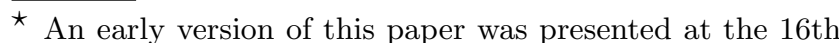
IFAC SYSID, Brussels, July 2012. This work was partly supported by the ITEA MODRIO project. Corresponding author M.B. Q.Z. is with INRIA, M.B. is with CNRS.

Email addresses: Qinghua.Zhang@inria.fr (Qinghua Zhang), Michele.Basseville@irisa.fr (Michèle Basseville).
}

application to some particular nonlinear systems. Another approach to dealing with nonlinear systems uses linearization along the actual or nominal trajectory of the monitored system. Such a linearization generally leads to linear time-varying (LTV) systems, whereas the more classical LTI approximation is usually related to the linearization around a single working point. It is thus clear that methods for FDI in LTV systems are much more powerful than their LTI counterparts. Finally, nonlinear control systems have been widely studied with the linear parameter varying (LPV) approach; see, e.g., $[7,37,44]$. Since a LPV system is essentially a particular LTV system, the FDI method proposed in this paper for general LTV systems is also valid for LPV systems.

FDI issues for LTV systems have been addressed using three main approaches known as fault detection filters, observers, and parity relations, as shortly recalled in the following. Even though the first and third approaches are known to be equivalent [48], and the third one may be seen as a particular case of the second one in the case of LTI systems (see for example [22]), it is useful to distinguish those three types of investigations.

The detection filter approach [38] has been investigated for LTV systems in [19, 34], and also in [14] by generalizing the least-squares derivation of the Kalman filter. A game theoretic approach to fault detection filter design has been proposed in [15], whereas an unknown input decoupled optimal filter for stochastic LTV systems with application to fault detection has been investigated 
in [11]. Based on the unknown input observer and decoupling technique of [27] and the innovation approach to filtering, the design of optimal filters for LTV systems with unknown inputs has been proposed and studied in [28]. More recently, the design of optimal stochastic fault detection filters for both LTI and LTV systems has been addressed in [13]. A filter-based approach to fault detection, estimation and tracking, and filter stability analysis have been studied in [33]. Designing a finite horizon $\mathcal{H}_{\infty}$ fault detection filter for discrete time LTV systems using projection and innovation analysis in Krein space has been the topic of $[56,58]$.

The design of adaptive observers for residual generation and the analysis of their convergence have been investigated in $[51,52,54]$. The design of adaptive observers for discrete time state affine systems has been recently addressed in [43]. Set-valued observers for solving the FDI problem for uncertain LTV systems have been proposed in [41, 42]. Fault detection for LTV systems has been addressed in the time domain and optimal solutions to different $\mathcal{H}_{-} / \mathcal{H}_{\infty}$ optimization problems have been provided in [36]. In [35], all those solutions have been shown to have the same observer structure. Finally, parity-based fault estimation for discrete LTV systems has been considered in [57].

A LPV gain scheduling approach to robust fault detection filter design was proposed in [9] and applied to actuators fault diagnosis in [10]. Another LPV gain scheduling method is proposed in [45] and applied to actuators fault diagnosis in $[46,47]$. Structured fault detection filters for LPV systems designed in [25] have been applied to fault detection in aircraft control surfaces servo-loop in [26]. Interval LPV observers proposed in [16] were applied to fault diagnosis for a wind turbine in [39].

In this paper, we address the FDI problem for LTV systems subject to parametric additive faults under weaker assumptions than usual, in particular regarding the number of required sensors and the stability of the monitored system. The proposed approach is statistical, by combining a generalized likelihood ratio (GLR) test with a recursive (Kalman) filter that cancels out the dynamics of the faults effects. To some extent, it has been inspired by the results reported in [24, 49, 50], but our approach addresses more general fault classes, with less assumption on the dynamics of the monitored system. More details about the relationship and differences between this paper and [24, 49, 50] are given in Subsections 2.2 and 2.4 and in the appendices.

The paper is organized as follows. The problem of additive faults in the state equation is stated in Section 2 and a simple filter is proposed in Section 3. The proposed FDI algorithm is described in Section 4. The case of additive faults in both state and output equations is considered in Section 5. Numerical results are presented in Section 6. Some conclusions are drawn in Section 7.

\section{Problem statement}

The considered fault-free stochastic multiple-input multiple-output (MIMO) LTV systems are of the form

$$
\left\{\begin{aligned}
X_{k+1} & =F_{k} X_{k}+G_{k} U_{k}+W_{k} \\
Y_{k} & =H_{k} X_{k}+J_{k} U_{k}+V_{k}
\end{aligned}\right.
$$

where $X_{k}$ is the $n$-dimensional state vector, $U_{k}$ is the $l$-dimensional input, $Y_{k}$ is the $p$-dimensional output, $F_{k}, G_{k}, H_{k}, J_{k}$ are bounded time-varying matrices of appropriate sizes, and $W_{k}$ and $V_{k}$ are two independent white Gaussian noise sequences with time-varying covariance matrices $Q_{k}$ and $R_{k}$, respectively. The initial state condition $X_{0}$ is assumed to be Gaussian with mean $\widehat{X}_{0}$ and covariance $P_{0}$. The matrix pair $\left(F_{k}, H_{k}\right)$ is assumed uniformly observable, and the matrix pair $\left(F_{k}, Q_{k}^{1 / 2}\right)$ uniformly controllable.

Before stating precisely the faults considered in this paper, let us first formulate the following general form of additive faults in the state equation:

$$
\left\{\begin{aligned}
X_{k+1} & =F_{k} X_{k}+G_{k} U_{k}+W_{k}+\Psi_{k} \theta_{k} \\
Y_{k} & =H_{k} X_{k}+J_{k} U_{k}+V_{k}
\end{aligned}\right.
$$

where the $m$-dimensional fault vector $\theta_{k}$ and the $n \times m$ fault profile matrix $\Psi_{k}$ are time-varying.

In (2), the term $\Psi_{k} \theta_{k}$ typically represents actuator faults.

Several particular and practical cases are discussed below. A particular case of similar additive faults in the output equation is considered in Subsection 2.4 and Section 5. For presentation simplicity and for comparison with existing results, the major part of this paper does not concern the faults in the output equation.

\subsection{Fault profiles and fault vectors}

As far as the fault profile matrix $\Psi_{k}$ and fault vector $\theta_{k}$ are concerned, two different modelling assumptions should be distinguished.

Constant fault profile and time-varying fault vector. A case often considered in the literature assumes a known constant fault profile matrix $\Psi$ and an unknown time-varying fault vector $\theta_{k}$; see e.g. [33]. In this case the number of sensors must be larger than or equal to the number of faults, namely $p \geq m$, in order to fully detect and isolate the $m$ faults (components of $\theta_{k}$ ).

Time-varying fault profile and constant fault vector. In contrast, in the present paper we consider the case where the matrix $\Psi_{k}$ is a possibly partly known time-varying fault profile and the $m$-dimensional vector $\theta$ is an unknown constant fault vector, that is:

$$
\left\{\begin{aligned}
X_{k+1} & =F_{k} X_{k}+G_{k} U_{k}+W_{k}+\Psi_{k} \theta \\
Y_{k} & =H_{k} X_{k}+J_{k} U_{k}+V_{k}
\end{aligned}\right.
$$


In this case, the condition on the number of sensors can be replaced by a persistent excitation condition as pointed out in Subsection 3.3. Important cases of actuator faults can be modelled with particular instances of the fault profile matrix $\Psi_{k}$ in (3) as described now.

For actuator biases, the time-varying matrix $\Psi_{k}$ is related to $G_{k}$. For example, if each actuator is possibly affected by a bias so that $U_{k}$ becomes $U_{k}+\theta$ (in this case $m=l)$, then: $G_{k}\left(U_{k}+\theta\right)=G_{k} U_{k}+\Psi_{k} \theta$ with $\Psi_{k}=G_{k}$. For faults affecting actuator gains, $\Psi_{k}$ is related to $U_{k}$. For example, if each actuator is possibly affected by a gain loss so that $U_{k}$ becomes $(\mathbf{I}-\operatorname{diag}(\theta)) U_{k}$ (again $m=$ $l$ ), where $\operatorname{diag}(\theta)$ is the diagonal matrix formed by the vector $\theta$ and $\mathbf{I}$ is the identity matrix of appropriate size, then: $G_{k}(\mathbf{I}-\operatorname{diag}(\theta)) U_{k}=G_{k} U_{k}+\Psi_{k} \theta$ where $\Psi_{k}=$ $-G_{k} \operatorname{diag}\left(U_{k}\right)$.

\subsection{Particular fault profiles}

It should be noted also that the fault profile matrix $\Psi_{k}$ may capture different fault occurrence speeds. In particular, it may involve an unknown fault onset time.

Step faults. As an example of faults occurring quickly, a step change in the actuator bias or gain could be represented by a jump in the parameter vector $\theta$ occuring at an unknown time. Since $\theta$ is assumed constant in the formulation (3), such a jump is represented by

$$
\Psi_{k}(r) \triangleq \widetilde{\Psi}_{k} \times \mathbb{1}_{\{k \geq r\}}
$$

where $\widetilde{\Psi}_{k}$ is the known fault profile, $r$ the unknown onset time of the jump, $\mathbb{1}_{\{\cdot\}}$ the indicator function, and the dependence of $\Psi_{k}$ on $r$ is made explicit in the notation $\Psi_{k}(r)$. In such a case, the estimation of the jump onset time $r$ might be of interest in addition to the detection of the fault itself.

For the above actuator bias and gain loss examples, if the considered faults occur suddenly at time $r$, then $\Psi_{k}$ is given by equation (4), with $\widetilde{\Psi}_{k}=G_{k}$ for biased actuators, and $\widetilde{\Psi}_{k}=-G_{k} \operatorname{diag}\left(U_{k}\right)$ for actuator gain losses.

Impulsive faults. These faults correspond to $\Psi_{k}=\delta_{r, k+1} \mathbf{I}$, where $\delta_{.,}$. is the Kronecker delta, and were considered in $[49,50]$. This case is also investigated in [24][Chap.9] with a similar approach. It should be noted that such an impulsive fault typically has a transient effect on the affected system due to its dynamic behavior. As shown in Appendix A, the approaches in $[49,50]$ and $[24][$ Chap.9] can be extended to the general fault profiles $\Psi_{k}$ considered here. However they implicitly assume the exponential stability of the state dynamics matrix $F_{k}$ of the monitored system, an assumption not required by the approach proposed in this paper.

\subsection{The problems to be solved}

Considering the fault-free model in (1) and the corresponding faulty system (3), and given a sequence of measurements $\left(U_{k}, Y_{k}\right)_{k}$, the problems addressed in this pa- per are to detect the presence of the monitored faults, to isolate them in terms of the non-zero components of the fault vector $\theta$, and possibly to estimate the fault onset time $r$ in the case of a fault profile as in (4). This formulation assumes that the nominal value of $\theta$ is zero. As it is straightforward to generalize to the case of a nonzero nominal value, we keep the case formulated above to simplify the presentation.

\subsection{Faults in both the state and output equations}

A more general case where additive faults may occur in both the state and output equations, namely

$$
\left\{\begin{aligned}
X_{k+1} & =F_{k} X_{k}+G_{k} U_{k}+W_{k}+\Psi_{k} \theta \\
Y_{k} & =H_{k} X_{k}+J_{k} U_{k}+V_{k}+\Phi_{k} \theta
\end{aligned}\right.
$$

where the $p \times m$ matrix $\Phi_{k}$ is another time-varying fault profile, can also be addressed with the approach proposed here, at the price of more complex computations. For the sake of notational simplicity, and also for the purpose of comparison with the results of $[49,50]$ and of [24][Chap.9], we first restrict the discussion to the fault model (3) and postpone to Section 5 the derivation of the solution for model (5). This more general case is covered neither in $[49,50]$ nor in [24], which consider only faults modelled by a particular term $\Psi_{k} \theta$ as in (3).

\section{Fault effect on the innovation}

In this section, we investigate the effect of the considered fault on the Kalman filter innovation sequence. As already shown in $[49,50]$ and [24][Chap.9] for particular instances of the fault model (3), this effect is additive; and moreover, the dynamic fault detection problem concerning the state-space system reduces to a static one concerning a regression model. The main contribution in this section is to exhibit a recursive and stable filter that cancels out the dynamics of the system and ensures reliable computation of the fault effect on the innovation by guaranteeing that all the variables involved in the algorithm are bounded, regardless of the stability of the monitored system. In contrast, the similar filters proposed in [49, 50] and [24][Chap.9] implicitly assumed the stability of the monitored system itself.

Let $\widehat{X}_{k}$ be the one-step ahead prediction of the state vector $X_{k}$, sometimes denoted as $\widehat{X}_{k \mid k-1}$ in the literature. This prediction is computed by ignoring the unknown fault term $\Psi_{k} \theta$, with the Kalman filter designed for the fault-free system (1). The computation is essentially made recursively through

$$
\widehat{X}_{k+1}=F_{k} \widehat{X}_{k}+G_{k} U_{k}+F_{k} K_{k}\left(Y_{k}-J_{k} U_{k}-H_{k} \widehat{X}_{k}\right)
$$

where $K_{k}$ is the Kalman gain computed as

$$
\begin{aligned}
K_{k} & =P_{k} H_{k}^{T} \Sigma_{k}^{-1} \\
P_{k+1} & =F_{k}\left(\mathbf{I}-K_{k} H_{k}\right) P_{k} F_{k}^{T}+Q_{k} \\
\Sigma_{k} & =H_{k} P_{k} H_{k}^{T}+R_{k} .
\end{aligned}
$$

Let $\widetilde{X}_{k}$ and $\varepsilon_{k}$ be the state and output prediction errors 
(the latter is also called the innovation):

$$
\begin{aligned}
\widetilde{X}_{k} & \triangleq X_{k}-\widehat{X}_{k} \\
\varepsilon_{k} & \triangleq Y_{k}-J_{k} U_{k}-H_{k} \widehat{X}_{k} .
\end{aligned}
$$

The behavior of these error sequences is analyzed next.

\subsection{Filtering the fault profile}

The innovation sequence $\varepsilon_{k}$ is the basic tool for FDI in this paper. The effect of the fault term $\Psi_{k} \theta$ on $\varepsilon_{k}$ is described in the following proposition.

Proposition 1 The innovation sequence $\varepsilon_{k}$ as defined in (10), of the Kalman filter (6) applied to the faulty system (3), is related to the fault parameter vector $\theta$ by

$$
\varepsilon_{k}=\varepsilon_{k}^{0}+H_{k} \Gamma_{k} \theta,
$$

where $\varepsilon_{k}^{0}$ denotes the innovation sequence of the same Kalman filter applied to the fault-free system (1), and $\Gamma_{k} \in \mathbb{R}^{n \times m}$ is recursively defined by

$$
\Gamma_{k+1}=F_{k}\left(\mathbf{I}-K_{k} H_{k}\right) \Gamma_{k}+\Psi_{k}, \quad \Gamma_{0}=0 .
$$

Remark 1 Though the Kalman filter (6) is designed for the fault-free system (1), it is always applied to the monitored system which is either fault-free (1) or faulty (3).

Proof of Proposition 1. In the case of the faulty system (3), it is straightforward to check that $\widetilde{X}_{k}$ and $\varepsilon_{k}$ satisfy the following recursions:

$$
\begin{aligned}
\widetilde{X}_{k+1} & =F_{k}\left(\mathbf{I}-K_{k} H_{k}\right) \widetilde{X}_{k}-F_{k} K_{k} V_{k}+W_{k}+\Psi_{k} \theta \\
\varepsilon_{k} & =H_{k} \widetilde{X}_{k}+V_{k}
\end{aligned}
$$

Define the linear combination of $\widetilde{X}_{k}$ and $\theta$ :

$$
\eta_{k} \triangleq \widetilde{X}_{k}-\Gamma_{k} \theta
$$

where $\Gamma_{k}$ is the $n \times m$ matrix recursively defined in (12). It then follows from (13) and (15) that:

$$
\begin{aligned}
\eta_{k+1} \triangleq & \widetilde{X}_{k+1}-\Gamma_{k+1} \theta \\
= & F_{k}\left(\mathbf{I}-K_{k} H_{k}\right)\left(\eta_{k}+\Gamma_{k} \theta\right)-F_{k} K_{k} V_{k}+W_{k} \\
& +\Psi_{k} \theta-\Gamma_{k+1} \theta \\
= & F_{k}\left(\mathbf{I}-K_{k} H_{k}\right) \eta_{k}-F_{k} K_{k} V_{k}+W_{k} \\
& +\left[F_{k}\left(\mathbf{I}-K_{k} H_{k}\right) \Gamma_{k}+\Psi_{k}-\Gamma_{k+1}\right] \theta
\end{aligned}
$$

The last term in (16) vanishes because of (12). Thus the recursion (16) for $\eta_{k}$ becomes:

$$
\eta_{k+1}=F_{k}\left(\mathbf{I}-K_{k} H_{k}\right) \eta_{k}-F_{k} K_{k} V_{k}+W_{k}
$$

On the other hand, in the fault-free case $\tilde{X}_{k}^{0}$ and $\varepsilon_{k}^{0}$ are also governed by equations in the forms of (13) and (14), but with $\theta=0$. Accordingly,

$$
\begin{aligned}
\tilde{X}_{k+1}^{0} & =F_{k}\left(\mathbf{I}-K_{k} H_{k}\right) \tilde{X}_{k}^{0}-F_{k} K_{k} V_{k}+W_{k} \\
\varepsilon_{k}^{0} & =H_{k} \widetilde{X}_{k}^{0}+V_{k}
\end{aligned}
$$

Since equation (17) governing $\eta_{k}$ is identical to equation (18) governing $\widetilde{X}_{k}^{0}$, an appropriate choice of the initial value $\widehat{X}_{0}$ so that $\eta_{0}=\widetilde{X}_{0}^{0}$ ensures that the equality

$$
\eta_{k}=\widetilde{X}_{k}^{0}
$$

holds for all $k \geq 0$. It follows from (14), (15), (20) that

$$
\begin{aligned}
\varepsilon_{k} & =H_{k}\left(\eta_{k}+\Gamma_{k} \theta\right)+V_{k} \\
& =H_{k} \eta_{k}+V_{k}+H_{k} \Gamma_{k} \theta \\
& =H_{k} \widetilde{X}_{k}^{0}+V_{k}+H_{k} \Gamma_{k} \theta
\end{aligned}
$$

The equality (11) is then proved from (21) and (19). This result shows that the effect of the fault in (3) on the innovation $\varepsilon_{k}$ is additive, as expected. Moreover that effect is governed by a matrix gain $\Gamma_{k}$ computed through (12), that depends on the time-varying fault profile matrix $\Psi_{k}$, but not on the fault vector $\theta$.

In the case of continuous time LTV systems, an equation similar to (11) has been derived in [55], through a general transformation for the design of adaptive observers. The derivation of (11) presented here follows a more straightforward approach.

\subsection{Stability and boundedness}

Under the assumptions of uniform observability and controllability made for the monitored system (1), the Kalman gain $K_{k}$ used in (6) is bounded and stabilizes the matrix $F_{k}\left(\mathbf{I}-K_{k} H_{k}\right)$ in the sense that the homogenous linear dynamic system $x_{k+1}=F_{k}\left(\mathbf{I}-K_{k} H_{k}\right) x_{k}$ is exponentially stable [32]. This implies that $\Gamma_{k}$, computed from the bounded $\Psi_{k}$ through the exponentially stable recursive equation (12), is also bounded. The boundedness of all the involved variables is thus guaranteed in the above numerical computations, regardless of the stability of the dynamics of system (1) characterized by $F_{k}$.

It should be noted that, in $[49,50,24]$, because the computations are based on recursive formulas that involve the state dynamics matrix $F_{k}$, the involved variables are not guaranteed to be bounded, unless the dynamics of the monitored system characterized by $F_{k}$ is itself exponentially stable. This fact is outlined in A.1 and numerically illustrated with the first example of Section 6 .

\subsection{Persistent excitation and required sensors number}

For detecting the faults represented by $\Psi_{k} \theta$ in (3) and estimating the unknown fault vector $\theta$ from (11) seen as a linear regression, in an off-line approach it is sufficient that the sum $\sum_{k} \Gamma_{k}^{T} H_{k}^{T} \Sigma_{k}^{-1} H_{k} \Gamma_{k}$ over the available data sample is strictly positive definite; here $\Sigma_{k}$ is the innovation covariance matrix (8). For the on-line approach of this paper, the following persistent excitation condition is assumed: there exist an integer $s>0$ and a real number $\alpha>0$ such that, for any $k \geq s$, the inequality

$$
\sum_{k=k-s+1}^{k} \Gamma_{k}^{T} H_{k}^{T} \Sigma_{k}^{-1} H_{k} \Gamma_{k} \geq \alpha \mathbf{I}
$$


holds, where $\mathbf{I}$ is the identity matrix of size $m$ (the dimension of $\theta$ ). Such a condition is typically assumed for on-line parameter estimation problems. This condition does not imply any hard requirement on the number of sensors, that is $p$, the dimension of $Y_{k}$, and can be satisfied when the number of sensors is smaller than $m$, the number of faults to be isolated, and in particular even with a single sensor $(p=1)$. When $p<m$, each term in the sum of $(22)$ is rank deficient. In this case the sum can still be positive definite if $\Gamma_{k}$, driven by the fault profile $\Psi_{k}$ through (12), varies sufficiently over the time.

\subsection{The transformed FDI problem}

Under the assumptions made in this paper, in the faultfree case the innovation $\varepsilon_{k}^{0}$ is a zero mean Gaussian white noise, whose covariance matrix, usually computed in the Kalman filter, is given in (8). Based on (11), where the (biased) innovation sequence $\varepsilon_{k}$ is computed in (10) with the aid of the Kalman filter (6), the considered FDI problem is thus transformed into the problem of monitoring a change in the mean of a white Gaussian sequence with known covariance matrix.

\section{Statistical tests for FDI}

Based on the main result of the previous section, summarized by (11), we propose to apply to the innovation sequence $\varepsilon_{k}$ the fault detection and estimation algorithm introduced in $[49,50]$ and further discussed in $[2,3]$. This algorithm is called generalized likelihood ratio (GLR) algorithm. The main idea is to perform a test between the fault-free model (1) - hypothesis $\mathbb{H}_{0}$ - and the faulty model (3) - hypothesis $\mathbb{H}_{1}$, respectively, using a likelihood ratio and replacing the unknown variables, namely the fault vector $\theta$ and possibly the fault onset time $r$, with their maximum likelihood estimates (MLE). In what follows, the GLR test is first formulated in the case of a fully known fault profile matrix $\Psi_{k}$, and then for a jump fault with unknown onset time.

\subsection{Fault detection - Known fault profile matrix}

Thanks to the additive effect of the fault in the linear regression equation (11), the fault-free and faulty hypotheses $\mathbb{H}_{0}$ and $\mathbb{H}_{1}$ can be formulated as

$$
\begin{aligned}
& \mathbb{H}_{0}: \varepsilon_{k} \sim \mathcal{N}\left(0, \Sigma_{k}\right) \\
& \mathbb{H}_{1}: \varepsilon_{k} \sim \mathcal{N}\left(H_{k} \Gamma_{k} \theta, \Sigma_{k}\right)
\end{aligned}
$$

where $\left(\varepsilon_{k}\right)_{k}$ is a white Gaussian innovation sequence with covariance matrix $\Sigma_{k}$ given in (8). The unknown value of $\theta$ under $\mathbb{H}_{1}$ is replaced with its MLE up to time $k$ :

$$
\begin{aligned}
\widehat{\theta}_{k} & \triangleq \arg \max _{\widetilde{\theta}} \ln p\left(\varepsilon_{1}, \ldots, \varepsilon_{k} \mid \theta=\widetilde{\theta}\right) \\
& =\arg \min _{\widetilde{\theta}} \sum_{j=1}^{k}\left(\varepsilon_{j}-H_{j} \Gamma_{j} \widetilde{\theta}\right)^{T} \Sigma_{j}^{-1}\left(\varepsilon_{j}-H_{j} \Gamma_{j} \widetilde{\theta}\right) \\
& =C_{k}^{-1} d_{k}
\end{aligned}
$$

and can be computed recursively as in (27)-(28), whereas

$$
\widehat{\theta}_{k}(r)=C_{k}^{-1}(r) d_{k}(r)
$$

and is compared to a threshold for deciding between $\mathbb{H}_{0}$ and $\mathbb{H}_{1}$. It is well known [3] that $l_{k}$ follows a $\chi^{2}$ distribution, centered under $\mathbb{H}_{0}$ and non centered under $\mathbb{H}_{1}$.

\subsection{Fault detection - Jump with unknown onset time}

When the dynamic fault profile is of jump-type as in (4), typically the fault onset time $r$ is unknown. In this case, the likelihood under $\mathbb{H}_{1}$ used in the GLR test should be maximized over the onset time $r$, in addition to the maximization with respect to the fault vector $\theta$. Let $k$ be the current time instant. For the $r$-dependent fault profile $\Psi_{k}(r)$ in (4), the filtered fault profile $\Gamma_{k}$ also depends on $r$ and is thus denoted by $\Gamma_{k}(r)$. Consequently equation (12) now writes:

$$
\Gamma_{k+1}(r)=F_{k}\left(\mathbf{I}-K_{k} H_{k}\right) \Gamma_{k}(r)+\Psi_{k}(r), \Gamma_{0}(r)=0
$$

Because $\Psi_{j}(r)=0$ for $j<r$, the fault term $\Psi_{k}(r) \theta$ does not affect the system and the matrix gain $\Gamma_{k}$ in (11) is not incremented before the jump onset time $r$. Thus the innovations $\varepsilon_{1}, \ldots, \varepsilon_{r}$ are independent of $\theta$, and the MLE of $\theta$ given in (24) becomes $\widehat{\theta}_{k}(r) \triangleq \arg \max _{\tilde{\theta}} \ln p\left(\varepsilon_{r+1}, \ldots, \varepsilon_{k} \mid \theta=\widetilde{\theta}\right)$. Accordingly, $C_{k}$ and $d_{k}$ now write:

$$
\begin{aligned}
& C_{k}(r) \triangleq \sum_{j=r+1}^{k} \Gamma_{j}^{T}(r) H_{j}^{T} \Sigma_{j}^{-1} H_{j} \Gamma_{j}(r) \\
& d_{k}(r) \triangleq \sum_{j=r+1}^{k} \Gamma_{j}^{T}(r) H_{j}^{T} \Sigma_{j}^{-1} \varepsilon_{j}
\end{aligned}
$$

and is computed for $r$ such that $0 \leq r \leq k-s$. The persistent excitation condition (22) ensures the invertibility of $C_{k}(r)$ computed with (32). 
At time $k$, for computing the likelihood for different values of $r \leq k-s$, the filtered fault profile $\Gamma_{k}(r)$ must be computed once for each value of $r \in\{1,2, \ldots, k-s\}$. This holds true for $C_{k}(r)$ and $d_{k}(r)$ as well.

Maximizing the likelihood ratio over the onset time $r$, the GLR statistics now writes:

$$
\begin{aligned}
l_{k} & =2 \max _{1 \leq r \leq k-s} \ln \frac{p\left(\varepsilon_{r+1}, \ldots, \varepsilon_{k} \mid \theta=\widehat{\theta}_{k}(r)\right)}{p\left(\varepsilon_{r+1}, \ldots, \varepsilon_{k} \mid \theta=0\right)} \\
& =\max _{1 \leq r \leq k-s} d_{k}^{T}(r) C_{k}^{-1}(r) d_{k}(r)
\end{aligned}
$$

and the estimated fault onset time is:

$$
\hat{r}_{k}=\arg \max _{1 \leq r \leq k-s} d_{k}^{T}(r) C_{k}^{-1}(r) d_{k}(r) .
$$

In practice the search of the maximum over $r$ is restricted within a moving time window of some size $w$, so that the computations of $\Gamma_{k}(r), C_{k}(r), d_{k}(r)$ are only made for

$$
r \in\{k-w+1, k-w+2, \ldots, k-s\} .
$$

\subsection{Fault isolation}

Assume that at time $k=k_{*}$ the GLR statistics $l_{k}$ has exceeded the chosen threshold so that a fault is detected. Fault isolation then consists in determining which (subsets of) components of the fault vector $\theta$ are responsible for the detected fault, namely which ones are non-zero.

In the case of a fully known fault profile $\Psi_{k}$, the whole sequence of innovations $\varepsilon_{1}, \ldots, \varepsilon_{k_{*}}$ is analyzed for fault isolation. For a jump fault with unknown onset time $r$ estimated as in (36), the sequence of innovations starting from the estimated jump time, namely $\varepsilon_{\hat{r}+1}, \ldots, \varepsilon_{k_{*}}$, is analyzed. In what follows, the latter case is considered without loss of generality, since the former case corresponds to $\hat{r}=1$. The vector $Z$ stacking those innovations on top of each other, namely:

$$
Z \triangleq\left[\begin{array}{c}
\varepsilon_{\hat{r}+1} \\
\vdots \\
\varepsilon_{k_{*}}
\end{array}\right]
$$

is a Gaussian vector:

$$
Z \sim \mathcal{N}(M \theta, R)
$$

with

$$
M=\left[\begin{array}{cc}
H_{\hat{r}+1} & \Gamma_{\hat{r}+1}(\hat{r}) \\
\vdots \\
H_{k} & \Gamma_{k_{*}}(\hat{r})
\end{array}\right], \quad R=\operatorname{diag}\left(\Sigma_{\hat{r}+1}, \ldots, \Sigma_{k_{*}}\right)
$$

Fault isolation then amounts to locating the non-zero components of $\theta$ in the mean $M \theta$ of the Gaussian vector $Z$. A typical approach consists in testing if a subset of the components of $\theta$ is different from zero, while considering the other components as nuisance information. By appropriately permuting the columns of $\Psi_{k}$ and the components of $\theta$, the vector $\theta$ is partitioned as:

$$
\theta=\left[\begin{array}{l}
\theta_{a} \\
\theta_{b}
\end{array}\right]
$$

where $\theta_{a}$ contains the assumed fault. The hypothesis $\theta_{a} \neq 0$ is tested against $\theta_{a}=0$ while considering $\theta_{b}$ as an unknown nuisance parameter to be statistically rejected [1]. This problem can be solved with the minmax test, that replaces the unknown $\theta_{b}$ with its least favorable value. This test runs as follows. Assuming the partition (40), the sensitivity matrix $M$ in (39), the Fisher information matrix $\mathbf{F} \triangleq M^{T} R^{-1} M$ and the normalized residual $\zeta \triangleq M^{T} R^{-1} Z$ are partitioned accordingly:

$$
\begin{aligned}
M & =\left[\begin{array}{ll}
M_{a} & M_{b}
\end{array}\right] \\
\mathbf{F} & =\left[\begin{array}{ll}
\mathbf{F}_{a a} & \mathbf{F}_{a b} \\
\mathbf{F}_{b a} & \mathbf{F}_{b b}
\end{array}\right] \triangleq\left[\begin{array}{ll}
M_{a}^{T} R^{-1} M_{a} & M_{a}^{T} R^{-1} M_{b} \\
M_{b}^{T} R^{-1} M_{a} & M_{b}^{T} R^{-1} M_{b}
\end{array}\right] \\
\zeta_{a} & =M_{a}^{T} R^{-1} Z, \quad \zeta_{b}=M_{b}^{T} R^{-1} Z
\end{aligned}
$$

and the statistics of the minmax test writes:

$$
l_{a}=\zeta_{a}^{* T} \mathbf{F}_{a}^{*-1} \zeta_{a}^{*}
$$

where $\zeta_{a}^{*}$ is the residual of the regression of $\zeta_{a}$ with respect to $\zeta_{b}$, namely: $\zeta_{a}^{*}=\zeta_{a}-\mathbf{F}_{a b} \mathbf{F}_{b b}^{-1} \zeta_{b}$ and $\mathbf{F}_{a}^{*}$ is its covariance: $\mathbf{F}_{a}^{*}=\mathbf{F}_{a a}-\mathbf{F}_{a b} \mathbf{F}_{b b}^{-1} \mathbf{F}_{b a}$. The statistics $l_{a}$ in (41) is then compared to some threshold in order to decide if $\theta_{a}=0$ or not. It is known that under the hypothesis $\theta_{a}=0$, regardless of the value of $\theta_{b}, l_{a}$ follows a centered $\chi^{2}$ distribution with a number of degrees of freedom equal to the dimension of $\theta_{a}$. This fact can serve as a guideline for the choice of the threshold $\lambda_{a}$ for $l_{a}$. Each decision made with the minmax test $l_{a}$ is subject to two types of errors:

Type I error: decide $\theta_{a} \neq 0$ while actually $\theta_{a}=0$

Type II error: decide $\theta_{a}=0$ while actually $\theta_{a} \neq 0$.

The threshold $\lambda_{a}$ is typically chosen for some desired probability of the type I error.

One procedure for isolating simultaneous faults consists in running simultaneously multiple nuisance rejectionbased isolation tests $l_{a_{1}}, \ldots, l_{a_{q}}$ corresponding to different partitions (40) of $\theta$, say $\left(\theta_{a_{1}}, \theta_{b_{1}}\right), \ldots,\left(\theta_{a_{q}}, \theta_{b_{q}}\right)$ [4]. The fault isolation decision is then made by comparing $l_{a_{1}}, \ldots, l_{a_{q}}$ to the corresponding thresholds $\lambda_{a_{1}}, \ldots, \lambda_{a_{q}}$ :

$$
l_{a_{1}} \lessgtr \lambda_{a_{1}}, \ldots, l_{a_{q}} \lessgtr \lambda_{a_{q}} .
$$

\section{Faults in both state and output equations}

As pointed out in Subsection 2.4, it is also possible to consider an additive fault term similar to $\Psi_{k} \theta$ in the output equation. Such a term, typically modelling sensor faults, can in principle be transformed into actuator faults through a state augmentation approach; see for example [38]. In order to avoid state augmentation, we generalize the method presented in this paper to the case where additive faults may appear in both the state and output equations. The case where such faults appear 
only in the output equation has been studied in [53]. The considered faulty system now writes

$$
\left\{\begin{aligned}
X_{k+1} & =F_{k} X_{k}+G_{k} U_{k}+W_{k}+\Psi_{k} \theta \\
Y_{k} & =H_{k} X_{k}+J_{k} U_{k}+V_{k}+\Phi_{k} \theta
\end{aligned}\right.
$$

where $\Psi_{k} \in \mathbb{R}^{n \times m}$ and $\Phi_{k} \in \mathbb{R}^{p \times m}$ are two known and bounded fault profile matrices, $\theta \in \mathbb{R}^{m}$ is the unknown fault vector, and the other quantities involved in this system are as in (1).

Though apparently the same fault parameter vector $\theta$ appears in both the state and the output equations, this formulation can also deal with independent faults in the two equations. Indeed by setting some columns of $\Psi_{k}$ to zeros, and the complementary columns of $\Phi_{k}$ to zeros, the faults affecting the two equations are then modelled by distinct components of the parameter vector $\theta$. It is also possible to keep some components of $\theta$ shared by $\Psi_{k}$ and $\Phi_{k}$. Therefore the formulation (44) is in fact general for coupled and non coupled faults in the state and the output equations. Note that this more general case is covered neither in $[49,50]$ nor in [24], which consider only faults modelled by a particular term $\Psi_{k} \theta$ as in (3). Like in Section 3, apply to system (44) the Kalman filter (6) which is designed by ignoring both fault terms $\Psi_{k} \theta$ and $\Phi_{k} \theta$. In this case the effect of the fault terms on the innovation $\varepsilon_{k}$ is described by the following proposition.

Proposition 2 The innovation sequence $\varepsilon_{k}$ as defined in (10), of the Kalman filter (6) applied to the faulty system (44), is related to the fault parameter vector $\theta$ by

$$
\varepsilon_{k}=\varepsilon_{k}^{0}+\left(H_{k} \Gamma_{k}+\Phi_{k}\right) \theta
$$

where $\varepsilon_{k}^{0}$ denotes the innovation sequence of the same Kalman filter applied to the fault-free system (1), and $\Gamma_{k} \in \mathbb{R}^{n \times m}$ is recursively defined by

$$
\Gamma_{k+1}=F_{k}\left(\mathbf{I}-K_{k} H_{k}\right) \Gamma_{k}+\Psi_{k}-F_{k} K_{k} \Phi_{k}, \Gamma_{0}=0 .
$$

Notice that, compared to equation (11) appearing in Proposition 1, the equation (45) involves an extra matrix $\Phi_{k}$, and the matrix $\Gamma_{k}$ now defined by (46) is different from the one in (12).

Proof of Proposition 2. Following the approach presented in Section 3 , let $\widetilde{X}_{k} \triangleq X_{k}-\widehat{X}_{k}$ be the state prediction error and define $\eta_{k} \triangleq \widetilde{X}_{k}-\Gamma_{k} \theta$, but now $\Gamma_{k}$ is defined as in (46). Like in the proof of Proposition 1, it can be easily checked that in this case

$\widetilde{X}_{k+1}=F_{k}\left(\mathbf{I}-K_{k} H_{k}\right) \widetilde{X}_{k}-F_{k} K_{k} V_{k}+W_{k}+\left(\Psi_{k}-F_{k} K_{k} \Phi_{k}\right) \theta$ and the new $\eta_{k}$ satisfies exactly (17) as before. Then

$$
\begin{aligned}
\varepsilon_{k} & =Y_{k}-J_{k} U_{k}-H_{k} \widehat{X}_{k} \\
& =H_{k} \widetilde{X}_{k}+V_{k}+\Phi_{k} \theta \\
& =H_{k} \eta_{k}+H_{k} \Gamma_{k} \theta+V_{k}+\Phi_{k} \theta
\end{aligned}
$$

Again like in the proof of Proposition 1, by appropriately choosing the initial value $\widehat{X}_{0}$, the resulting $\eta_{k}$ satisfies

$$
\varepsilon_{k}^{0}=H_{k} \eta_{k}+V_{k}
$$

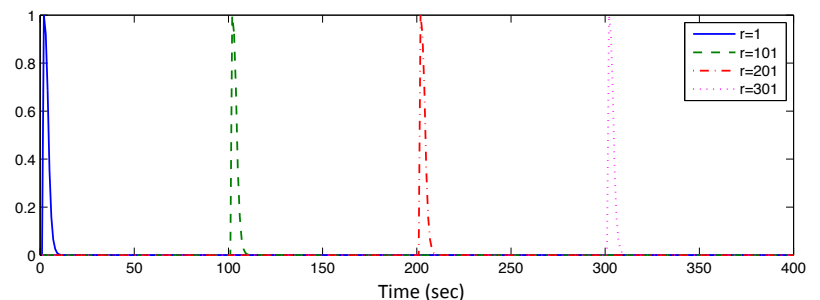

Figure 1. The spectral norm of $\Gamma_{k}$ for $r=1,101,201,301$.

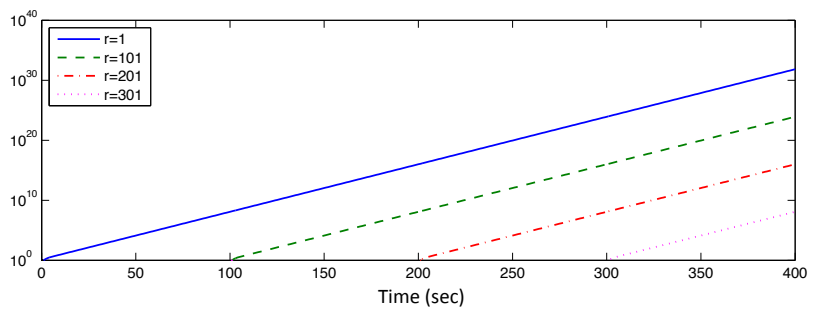

Figure 2. The spectral norm of $\alpha_{k}(r)$ for $r=1,101,201,301$.

where $\varepsilon_{k}^{0}$ is the unbiased Kalman innovation in (19). The equality in (45) is then obtained from (49) and (50). Due to the similarity between the results of Proposition 1 and Proposition 2, after the computations of $\varepsilon_{k}$ and $\Gamma_{k}$, the algorithms presented in Section 4 apply as before, but $H_{k} \Gamma_{k}$ is now replaced with $\left(H_{k} \Gamma_{k}+\Phi_{k}\right)$.

Let us end this subsection by a note on algorithm stability. Under uniform observability and uniform controllability conditions, the Kalman gain $K_{k}$ is bounded and ensures the exponential stability of the matrix LTV system (46) characterized by the time varying matrix $F_{k}\left(\mathbf{I}-K_{k} H_{k}\right)$. The assumed boundedness of $F_{k}, \Psi_{k}$ and $\Phi_{k}$ then imply the boundedness of $\Gamma_{k}$, ensuring reliable numerical computations.

\section{Numerical examples}

In order to illustrate the relevance of the proposed approach to FDI in LTV systems, in this section we report on the numerical results obtained on two examples: a simulated example in order to illustrate the stability issue discussed above, and a more realistic one borrowed from the literature in order to present and thoroughly discuss the numerical results obtained for FDI.

\subsection{A simple example illustrating the stability issue}

The purpose of the first example is to illustrate an important difference between the method presented in this paper and the one proposed in [49, 50] [24][Chap. 9]: in the former case all the involved variables have bounded values, whereas in the latter case some variables may tend to infinity if the monitored system is unstable. Consider the state space system modeled by

$$
\begin{aligned}
X_{k+1} & =\left[\begin{array}{cc}
0.5 & 1 \\
0 & 1.2
\end{array}\right] X_{k}+\left[\begin{array}{l}
0 \\
1
\end{array}\right] U_{k}+W_{k}+\delta_{r, k+1} \theta \\
Y_{k} & =\left[\begin{array}{ll}
1 & 0
\end{array}\right] X_{k}+V_{k}
\end{aligned}
$$



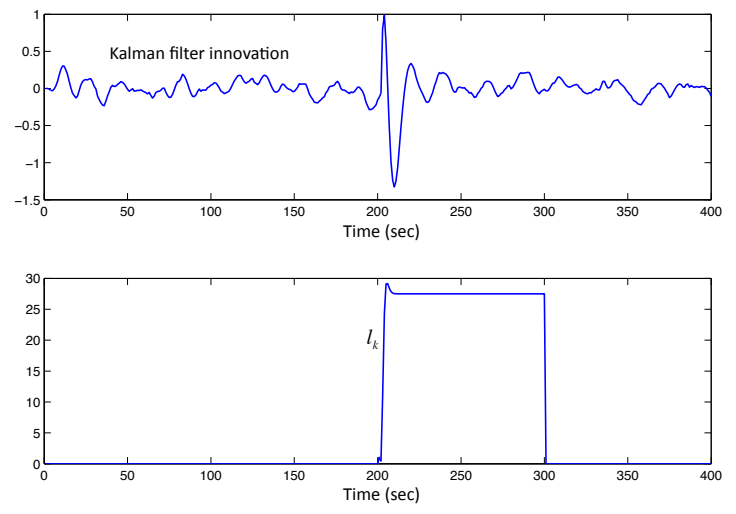

Figure 3. Kalman filter innovation (Top) and GLR statistics $l_{k}$ (Bottom) for the impulsive fault occurring at $k=201$ with $\theta=[4,0]^{T}$. GLR search window size $w=100$.
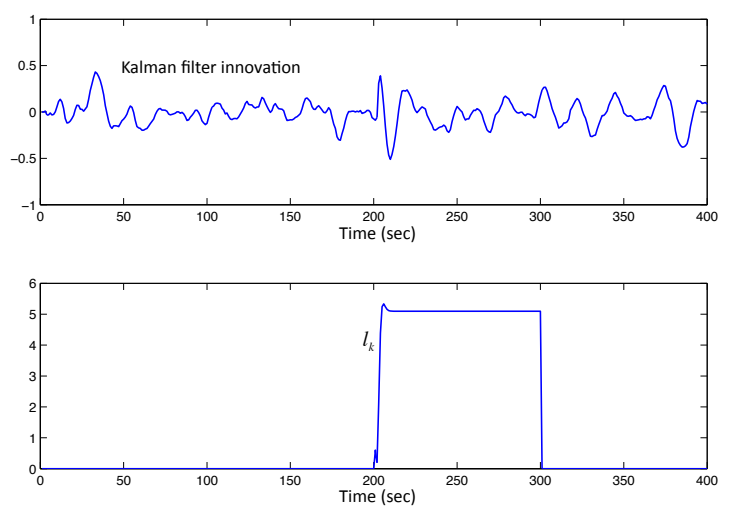

Figure 4. Kalman filter innovation (Top) and GLR statistics $l_{k}$ (Bottom) for the impulsive fault occurring at $k=201$ with $\theta=[1.5,0]^{T}$. GLR search window size $w=100$.

where $X_{k} \in \mathbb{R}^{2}, U_{k} \in \mathbb{R}, Y_{k} \in \mathbb{R}$, the noises $W_{k} \in$ $\mathbb{R}^{2}, V_{k} \in \mathbb{R}$, and the fault vector $\theta \in \mathbb{R}^{2}$. The sampling period is $T_{s}=1 \mathrm{sec}$. The noises covariances are $Q_{k}=0.0025 \times \mathbf{I}$ and $R_{k}=0.0025$. This LTI example is chosen for its easy stability analysis. A more complex LTV (LPV) system is considered in the next subsection.

One of the eigenvalues of the state dynamics matrix is 1.2808 , hence the system is unstable. It is stabilized by a PI controller represented by the transfer function $C(z)=0.209+0.0011 / z-1$. The input $U_{k}$ of the system is given by the PI controller, that ensures that the state of the system remains bounded. The setpoint of the closed loop system is equal to 1 .

The main issue in this example is the stability of the filter (12) computing $\Gamma_{k}$, given that the state dynamics matrix $F_{k}$ has an unstable eigenvalue. Remind that, in theory, the Kalman gain $K_{k}$ ensures that the recursion in (12) is exponentially stable [32]. Thus $\Gamma_{k}$ computed with (12) driven by any bounded $\Psi_{k}$ is bounded. To experimentally check this boundedness in the present example where $\Psi_{k}=\delta_{r, k+1} \mathbf{I}$, the computation of the sequence $\Gamma_{k}$ is repeated four times, each with $r=1,101,201$ or 301 . The spectral norm of $\Gamma_{k}$ is plotted in Figure 1 for the four sequences. The results show that in all the cases the norm of $\Gamma_{k}$ remains under the value of 1 .

The counterpart of $\Gamma_{k}$ in the method of $[49,50]$ is $\alpha_{k}(r)$ defined by the recursion (A.6). It is also computed for each value of $r=1,101,201,301$. The spectral norm of $\alpha_{k}(r)$ is plotted in the logarithmic scale in Figure 2 for the four cases. These curves appear linearly increasing with time, indicating that the spectral norms increase exponentially. These results confirm that $\alpha_{k}(r)$ may tend to infinity when the considered system is unstable.

To see the ability of the proposed fault detection method for such an unstable system, two cases corresponding to two different values of $\theta$ are simulated. In the first case $\theta=[4,0]^{T}$ and the simulated fault occurs at $k=201$. The Kalman innovation $\varepsilon_{k}$ and GLR statistics $l_{k}$ in (35) are illustrated in Figure 3. As the simulated impulsive fault disappears after $k=r-1\left(\delta_{r, k+1}=0\right.$ for $\left.k>r-1\right)$, its effect on the innovation sequence is visible only during a short time interval after $k=201$. In contrast, the GLR statistics $l_{k}$ raises significantly during about $100 \mathrm{sec}$. In fact, the GLR search window size chosen in this example is $w=100$. For $k>301$, the impulsive fault occurring at $k=201$ gets out of the search window, then the effect of the fault on $l_{k}$ disappears. It is clearly easier to detect the fault by thresholding the GLR statistics $l_{k}$ than directly thresholding the Kalman innovation $\varepsilon_{k}$.

The second case, with a smaller fault magnitude corresponding to $\theta=[1.5,0]^{T}$, also occurring at $k=201$, is presented in Figure 4. In this case, the effect of the impulsive fault is hardly visible on the Kalman filter innovation sequence. Nevertheless, this fault can be detected without ambiguity by thresholding the GLR statistics $l_{k}$.

\subsection{Leakage detection in a gas transportation network}

As an illustration of the relevance of the proposed FDI approach, we now consider an application example borrowed from [37] on leakage detection in natural gas transportation networks. Leakage detection and localization is a crucial issue for pipeline operators, and especially for high pressure natural gas transportation networks, since an early warning of and proper reaction to a leakage allow effective risk minimization. Many gas leakage detection methods exist, ranging from manual inspections to model-based approaches using pressure or mass flow signals recorded mostly at the two ends of the pipeline. A major difficulty in detecting leakage from massflow balance equations is caused by the presence of a significant amount of noises in the measurements, hence the interest of the proposed statistical approach.

Model and simulated faults. In [37], a LPV model of the gas dynamics in a gas transportation network is established through a system identification approach. 
The resulting discrete time LPV model writes:

$\left\{\begin{aligned} X_{k+1} & =\left(F_{0}+F_{p} p_{k}\right) X_{k}+\left(G_{0}+G_{p} p_{k}\right) U_{k}+W_{k} \\ Y_{k} & =\left(H_{0}+H_{p} p_{k}\right) X_{k}+\left(J_{0}+J_{p} p_{k}\right) U_{k}+V_{k}\end{aligned}\right.$

where $U_{k} \in \mathbb{R}$ is the input mass flow, $Y_{k} \in \mathbb{R}$ is the output mass flow, $X_{k} \in \mathbb{R}^{2}$ is composed of the mass flow and the pressure drop within the first section of the modeled pipe, and $p_{k} \in \mathbb{R}$ is the scheduling parameter (pressure pattern). The parameter values contained in $F_{0}, F_{p}, G_{0}, G_{p}, H_{0}, H_{p}, J_{0}, J_{p}$ are detailed in [37].

In this paper two types of faults are considered. First, faults are simulated as leakages at the input end of the considered pipe. They are modelled as depression in the first components $G_{0}(1)$ and $G_{p}(1)$ of $G_{0}$ and $G_{p}$. Second, a leakage is simulated at the output end of the pipe, by adding a bias into the output equation of (53). This second case is similar to the one considered in [37].

In the simulations presented below, the input data $U_{k}$ was provided by the authors of [37], corresponding to a record of $1000 \mathrm{~min}$ of the second day as presented in [37]. The sampling period is $2 \mathrm{~min}$. The output data are simulated with the LPV model (53) by adding the simulated fault into the state or the output equation. The sequences $W_{k}$ and $V_{k}$ are generated as two independent Gaussian white noises with constant covariance matri$\operatorname{ces} Q=\left[\begin{array}{cc}100.2766 & -66.1165 \\ -66.1165 & 43.5933\end{array}\right], R=3.0273 \times 10^{5}$.

Leakages at the input end. At the input end of the considered pipe, faults are simulated as depression of the first components of the vectors $G_{0}$ and $G_{p}$, respectively denoted by $G_{0}(1)$ and $G_{p}(1)$. Each of the simulated faults, affecting either $G_{0}(1)$ or $G_{p}(1)$, occurs at the 600-th min as a jump of the concerned parameter. The nominal and changed values of $G_{0}(1)$ and $G_{p}(1)$ are displayed in Table 1 . These changes have been chosen small enough so that their effects are hardly visible on the noisy Kalman innovation often used as a residual for fault detection. For the faults considered in this example, the matrix $\Psi_{k}$ and parameter vector $\theta$ in (3) take the form

$$
\Psi=\left[\begin{array}{cc}
U_{k} & p_{k} U_{k} \\
0 & 0
\end{array}\right], \theta=\left[\begin{array}{c}
\tilde{G}_{0}(1) \\
0
\end{array}\right] \text { or } \theta=\left[\begin{array}{c}
0 \\
\tilde{G}_{p}(1)
\end{array}\right]
$$

where $\tilde{G}_{0}(1)$ and $\tilde{G}_{p}(1)$ are respectively the increments of $G_{0}(1), G_{p}(1)$ as in Table 1.

Table 1 The simulated faults.

\begin{tabular}{|l|ll|}
\hline Nominal values & Changed values & \\
\hline$G_{0}(1)=-7.8297 \times 10^{-4}$ & $G_{0}(1)-2 \times 10^{-5}$ & (Fault 1) \\
$G_{p}(1)=+3.8290 \times 10^{-5}$ & $G_{p}(1)-1 \times 10^{-5}$ & (Fault 2) \\
\hline
\end{tabular}

In the results reported below, the computations of $\Gamma_{k}(r), C_{k}(r), d_{k}(r)$ in (12), (25) and (26), respectively,
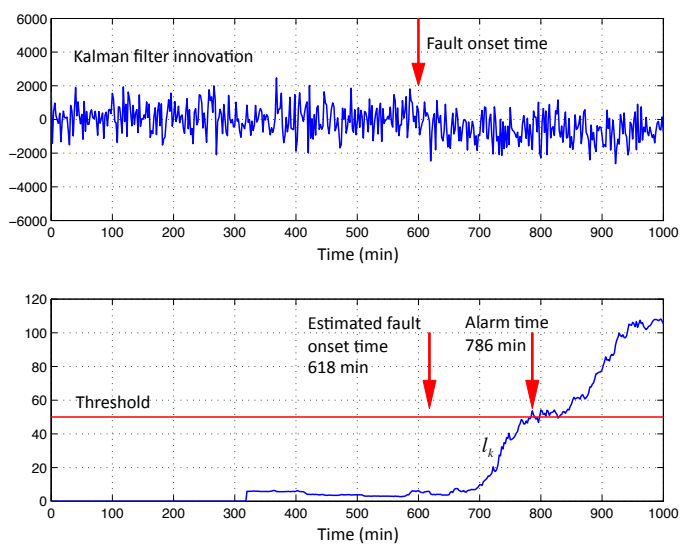

Figure 5. Fault detection - Fault 1 with $\tilde{G}_{0}=-2 \times 10^{-5}$.
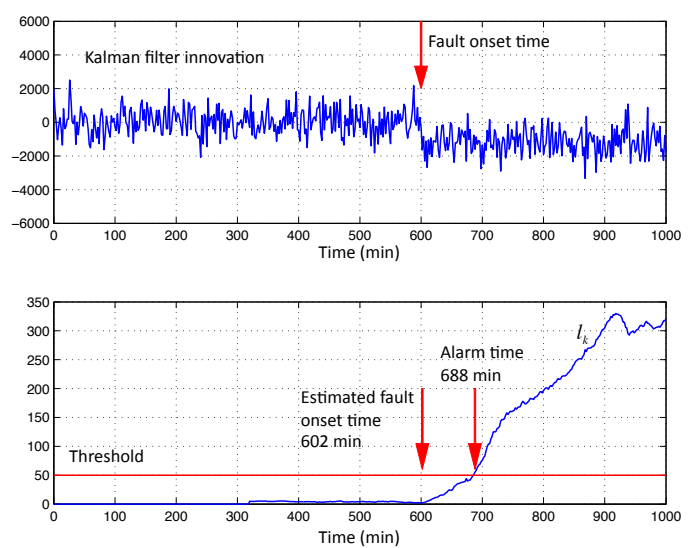

Figure 6. Fault detection - Fault 1 with $\tilde{G}_{0}=-4 \times 10^{-5}$, doubled with respect to the case of Figure 5 . The effect of the fault on the Kalman innovation is more visible, and the GLR test statistics $l_{k}$ grows more rapidly.

necessary for computing the test (35) and the fault onset time estimate (36), are performed within a moving search window of fixed size $w=160$ samples $(320 \mathrm{~min})$. See Section 4.2 for the discussion about this window. The GLR test is disabled during the first $320 \mathrm{~min}$ while waiting for the search window being fully filled.

Figure 5 (Top) displays the innovation of the Kalman filter computed on data generated with fault 1 . It appears that the effect of the fault, drowned in the noisy innovation sequence, is hardly visible. Figure 5 (Bottom) displays the behavior of the test statistics $l_{k}$ as in (35). With the detection threshold equal to 50, the alarm is raised at the 786 -th min. The estimated fault onset time, given by (36), is the 618-th min.

In Figure 5 (Bottom), the curve of the GLR test statistics $l_{k}$ as defined in (35) can clearly detect the fault with a threshold chosen within a quite large range (from 20 to 100). For the threshold choice as shown in Figure 5, the large detection delay of $186 \mathrm{~min}$ is because of the 
small magnitude of the simulated fault whose effect is hardly visible in the noisy Kalman innovation sequence. To observe the influence of the fault magnitude on the detection delay, in a second simulation the magnitude of the fault is doubled, with $\tilde{G}_{0}=-4 \times 10^{-5}$ instead of the previously used value $-2 \times 10^{-5}$, and the result is shown in Figure 6. In this case the effect of the fault is more visible on the Kalman innovation sequence, but it remains delicate to apply a threshold to the innovation sequence in order to detect this fault. The GLR test statistics $l_{k}$ grows more rapidly in this case. With the same threshold as before, the detection delay is $88 \mathrm{~min}$. This example confirms that a higher fault magnitude implies a smaller detection delay. The detection delay could also be reduced by lowering the threshold, but at the price of an increased false alarm rate.

Each of the results shown in Figures 5 and 6 corresponds to one simulation with a random realization of the simulated noises. To gain insights about the statistical behavior of the algorithm, for each of the simulated faults with magnitudes given in Table 1, 1000 random realizations are repeated. The histograms of the detection delays and of the fault onset time estimation errors are displayed in Figure 7, with fault 1 on the left side and fault 2 on the right side. In 7 out of the 2000 realizations, the GLR statistics $l_{k}$ exceeded the chosen threshold before the occurrence of the simulated fault (false alarms). Such results depend on the magnitudes of the simulated faults, and here the examples have been chosen so that they cannot be trivially detected by thresholding the Kalman innovation sequence.
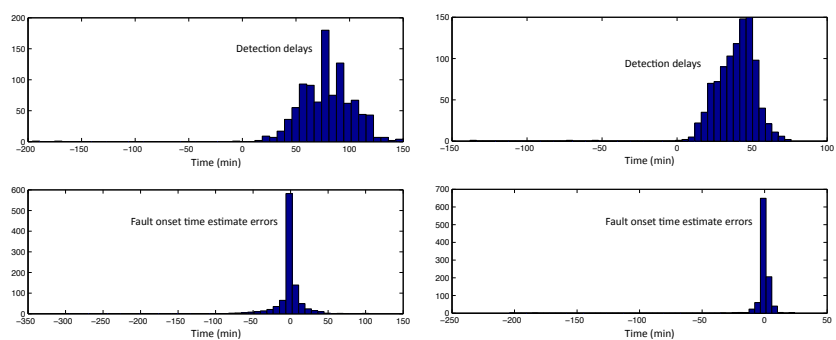

Figure 7. Histograms of detection delays (Top) and onset time estimation errors (Bottom), fault 1 (Left) and 2 (Right).

After fault detection (when the GLR test $l_{k}$ has exceeded the chosen threshold), fault isolation is performed with two parallel minmax tests in the form of (41), one focusing on fault 1 and the other on fault 2. The histograms of the results based on 1000 realizations for each of the two simulated faults are displayed in Figure 8.

On the left-hand side of Figure 8 the results for simulated fault 1 are shown, with the minmax test focussing on fault 1 (Top) and the minmax test focussing on fault 2 (Bottom). The vertical red lines indicate the threshold of 6.6349 corresponding to the $1 \%$ theoretic rate of type I error for each minmax test, as defined in (42). Over the 1000 realizations simulating fault 1 , no type II error is encountered, and the rate of type I error is $1.7 \%$.
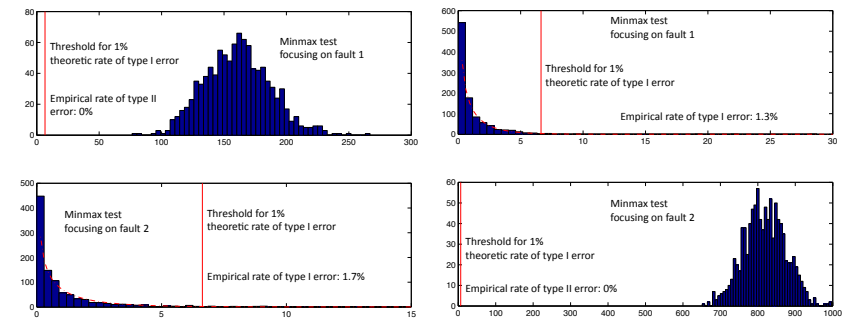

Figure 8. Histograms of fault isolation minmax tests. Fault 1 (Left), fault 2 (Right). Minmax tests focusing on fault 1 (Top) and on fault 2 (Bottom). The dashed red lines correspond to the theoretic centered $\chi^{2}(1)$ probability density function rescaled to the scale of the histograms. See (42) for the definition of type I and type II errors.

Similar results are shown on the right-hand side of Figure 8 for the case of fault 2. Empirically over the 1000 realizations simulating fault 2 , the rate of type I error is $1.3 \%$, and the rate of type II error is $0 \%$.

In these results based on 1000 realizations for each of the two simulated faults, the histograms of each minmax test when the monitored fault is absent is in good agreement with the theoretic $\chi^{2}(2)$ distribution as illustrated by the dashed red lines in Figure 8. In these realizations the empirical rates of type II error are equal to zero, despite the small magnitudes of the simulated faults.
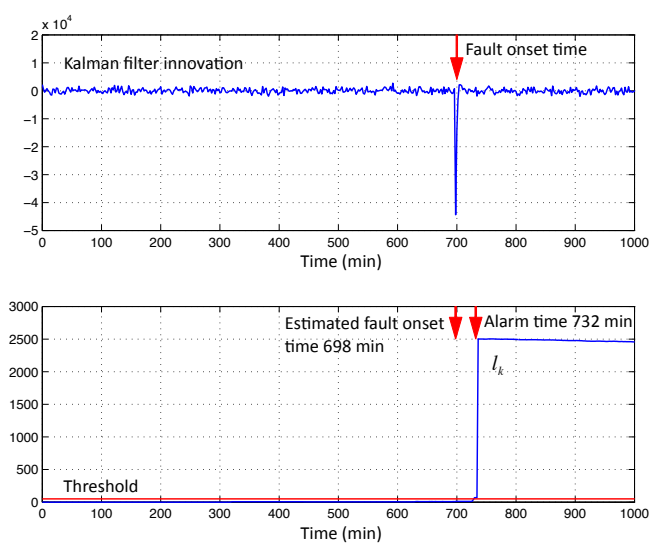

Figure 9. Fault detection - Leakage at the output end. After the occurrence of the simulated leakage, the innovation sequence drops suddenly, before going back to a level close to the average before the fault. The GLR test statistics $l_{k}$ increases rapidly and stays at a level of about 50 times the threshold. The threshold value is 50 as above.

Leakage at the output end. To simulate a leakage fault at the output end of the considered pipe, a constant bias of $-43980 \mathrm{Nm}^{3}$, corresponding to $10 \%$ of the average output mass flow, is added to the output equation of (53) at the 700-th min. This case is similar to the one considered in [37]. For this example, $\Phi_{k}=1$ after the (unknown) jump time.

Figure 9 (Top) displays the innovation of the Kalman filter computed on data generated with the simulated leak- 
age. After the occurrence of the simulated leakage, the innovation sequence drops suddenly, before going back to a level close to the average before the simulated fault, though the simulated fault is persistent in the system. In this case it is possible to detect the fault based on the transient behavior of the Kalman innovation sequence. However, if a smooth fault profile was simulated, the transient drop of the innovation sequence would be less obvious. It is more reliable to make detections which do not solely rely on the transient effect of a fault. This case has been successfully addressed in [37]. As an alternative solution, the method presented in this paper is applied. The GLR test statistics $l_{k}$ is plotted in Figure 9 (Bottom). After the occurrence of the simulated fault, the value of $l_{k}$ exceeds rapidly the chosen threshold and stays at a quite high level (about 50 times the threshold). This behavior of $l_{k}$ is to be compared with that of the innovation sequence, on which the effect of the fault is clearly visible only during a short transient interval.

\section{Conclusion}

The FDI problem for LTV systems has been addressed using a statistical approach combining a recursive filter that cancels out the fault dynamics and a GLR test. The proposed algorithm handles additive parametric faults, with weaker assumptions than usual on the number of required sensors. Numerical results obtained on a simulated example confirm the stability of the proposed filter even for an unstable system, an advantage w.r.t. $[49,50,24]$, and the capability of the detection algorithm to detect additive faults hardly visible on the innovation sequence. Numerical results for leakage detection in a gas transportation network further illustrate the effectiveness of the proposed FDI method, both for detecting relatively small faults and for isolating the components of the fault vector.

The online computational cost of the proposed method can be evaluated separately for the Kalman filter-based residual generation part and the GLR test part. For the first part, in addition to the well known numerically efficient Kalman filter, the computational cost is mainly for the computation of the matrix $\Gamma_{k}$ through (12). At each time instant $k$, the number of floating point operations (FLOP) is of the order of $O\left(n^{3}\right)+O\left(n^{2} p\right)+O\left(n^{2} m\right)$, where $n$ is the dimension of the state vector, $p$ is the number of outputs and $m$ is the number of components in the fault parameter vector $\theta$. For the second part, in the case of known fault profile (Subsection 4.1), at each time instant $k$, the number of FLOP is of the order of $O\left(n m^{2}\right)+O(n m p)+O\left(m p^{2}\right)+O\left(m^{3}\right)+O\left(p^{3}\right)$. In the case of jump fault with unknown onset time (Subsection 4.2), this cost is roughly multiplied by the size $w$ of the moving search window. Overall the online computational cost is roughly proportional to the third power of the sizes of the variables involved in the considered system, possibly multiplied by the window size when the GLR test is maximized within a moving search window. This result is essentially due to the fact that the nu- merical computations mainly involve matrix multiplications and inversions. After an alarm raised by the GLR test, the computational cost for fault isolation should be added, but this computation is not made for every time instant $k$. Each minmax test for fault isolation costs $O\left(m^{3}\right)+O\left(m^{2}(p w)\right)+O\left(m(p w)^{2}\right)+O\left((p w)^{3}\right)$ FLOP.

The assumption of parametric faults may be considered a drawback in practice, as it is less flexible than the more versatile arbitrary unknown fault profile assumption considered in other publications. On the other hand, it is also this parametric fault assumption that weakens the requirement on the number of sensors. The possibility of assuming parametric faults depends on the available prior knowledge about the considered faults. Even in the case of over-equipped sensors, a more detailed fault model can lead to more efficient FDI algorithms. Another drawback related to parametric faults is the additional assumption about persistent excitation.

Future investigations include experiments on other simulated and real cases to confirm the performances of the proposed algorithm, and the design and analysis of FDI algorithms for LTV systems affected by faults known with different levels of a priori information.

\section{A Revisiting the Willsky-Jones algorithm}

In this Appendix, we first recall the Willsky-Jones algorithm as proposed in $[49,50]$, then we investigate the relationship and the difference between this algorithm and the one proposed in the present paper.

\section{A.1 The Willsky-Jones fault model and algorithm}

The fault model considered in $[49,50]$ is:

$$
\left\{\begin{aligned}
X_{k+1} & =F_{k} X_{k}+G_{k} U_{k}+W_{k}+\delta_{r, k+1} \theta \\
Y_{k} & =H_{k} X_{k}+J_{k} U_{k}+V_{k}
\end{aligned}\right.
$$

Here $\delta_{r, k+1} \theta$ represents a possible jump in one or more of the state variables, $r>0$ is an integer with finite value if a jump occurs and $+\infty$ if there is no jump. Note that the fault vector $\theta$ is assumed to be deterministic and to have the same dimension as the state $X$.

Effect of the fault on the innovation. Let us recall the analysis of the fault effect on the innovation made in $[49,50]$. This analysis follows an approach different from the one proposed in Section 3 of the present paper. Because of the additive nature of the fault, the state, the state estimate and the output prediction error (innovation) can be decomposed as the sum of two parts: one solely due to the fault-free assumption (denoted with an exponent " 0 ") and one due to the additive fault. The matrices $\alpha_{k}(r), \xi_{k}(r), \rho_{k}(r)$ are defined accordingly as:

$$
\begin{aligned}
X_{k} & =X_{k}^{0}+\alpha_{k}(r) \theta \\
\widehat{X}_{k \mid k} & =\widehat{X}_{k \mid k}^{0}+\xi_{k}(r) \theta \\
\varepsilon_{k} & =\varepsilon_{k}^{0}+\rho_{k}(r) \theta
\end{aligned}
$$


where $\alpha_{k}(r), \xi_{k+1}(r), \rho_{k}(r)$ are non-zero only for $k \geq r$, the innovation $\varepsilon_{k}$ is defined by (10), and $\widehat{X}_{k \mid k}$ is the updated state estimate related to the one-step ahead prediction $\widehat{X}_{k} \triangleq \widehat{X}_{k \mid k-1}$ by

$$
\widehat{X}_{k \mid k}=\widehat{X}_{k}+K_{k} \varepsilon_{k}
$$

with $K_{k}$ being the Kalman gain (7). Based on (A.1), (A.5), (6) and (10), $\alpha_{k}(r), \xi_{k+1}(r), \rho_{k}(r)$ satisfy:

$$
\begin{aligned}
\alpha_{k+1}(r) & =F_{k} \alpha_{k}(r) \\
\xi_{k+1}(r) & =F_{k+1} \xi_{k}(r)+K_{k+1} \rho_{k+1}(r) \\
\rho_{k+1}(r) & =H_{k+1}\left(\alpha_{k+1}(r)-F_{k+1} \xi_{k}(r)\right)
\end{aligned}
$$

with the initial conditions $\alpha_{k}(k)=\mathbf{I}, \xi_{k}(k)=K_{k} H_{k}, \rho_{k}(k)=$ $H_{k}$. Note that $\alpha_{k}(r)$ and $\xi_{k}(r)$ computed through (A.6)(A.7) may not be bounded unless the homogeneous system $x_{k+1}=F_{k} x_{k}$ is exponentially stable. Remark that the algorithm in $[49,50]$ did rely on the possibly unbounded (A.6)-(A.7); see (A3)-(A4) in [50].

FDI algorithm. From (A.4) which is similar to (11), the GLR test, the MLE of $\theta$ and the estimated fault onset time write as in (35), (34), (36), respectively, with $C_{k}(r)$ and $d_{k}(r)$ defined as in (32)-(33) (with $H_{j} \Gamma_{j}(r)$ replaced by $\left.\rho_{j}(r)\right)$ and computed recursively as in (27)-(28).

\section{A.2 The Willsky-Jones algorithm revisited}

We now modify the Willsky-Jones algorithm to avoid the possibly unbounded computations in the original algorithm recalled in A.1. The key element is replacing in (A.3) the updated state estimate $\widehat{X}_{k \mid k}$ by the one-step ahead prediction $\widehat{X}_{k} \triangleq \widehat{X}_{k \mid k-1}$. This modification is clearly inspired by the new approach proposed in Section 3. The fault model considered here is still model (A.1).

Effect of the fault on the innovation. This effect is computed in the following manner. Because of the additive nature of the fault, the state, the one-step ahead prediction of the state and the output prediction error can be decomposed as the addition of two parts : one part solely due to the fault-free assumption and one part due to the additive fault. The matrices $\alpha_{k}(r), \beta_{k}(r), \rho_{k}(r)$ are defined accordingly as:

$$
\begin{aligned}
X_{k} & =X_{k}^{0}+\alpha_{k}(r) \theta \\
\widehat{X}_{k} & =\widehat{X}_{k}^{0}+\beta_{k}(r) \theta \\
\varepsilon_{k} & =\varepsilon_{k}^{0}+\rho_{k}(r) \theta
\end{aligned}
$$

where $\alpha_{k}(r), \beta_{k+1}(r), \rho_{k}(r)$ are non-zero only if $k \geq r$. Based on (A.1), (6) and (10), $\alpha_{k}(r), \beta_{k+1}(r), \rho_{k}(r)$ satisfy:

$$
\begin{aligned}
\alpha_{k+1}(r) & =F_{k} \alpha_{k}(r) \\
\beta_{k+1}(r) & =F_{k} \beta_{k}(r)+F_{k} K_{k} \rho_{k}(r) \\
\rho_{k}(r) & =H_{k}\left(\alpha_{k}(r)-\beta_{k}(r)\right)
\end{aligned}
$$

with the initial conditions: $\alpha_{k}(k)=\mathbf{I}, \beta_{k+1}(k)=$ $F_{k} K_{k} H_{k}, \rho_{k}(k)=H_{k}$. Note the difference between (A.14) and (A.8). Now, if we define

$$
\gamma_{k}(r) \triangleq \alpha_{k}(r)-\beta_{k}(r)
$$

then the relation (A.14) writes

$$
\rho_{k}(r)=H_{k} \gamma_{k}(r)
$$

and moreover, the two recursions (A.12) and (A.13) can be replaced by the single one

$$
\gamma_{k+1}(r)=F_{k}\left(\mathbf{I}-K_{k} H_{k}\right) \gamma_{k}(r)
$$

with the initial condition $\gamma_{k}(k)=\mathbf{I}$. This result is indeed similar to (11) for an impulsive fault as in model (A.1). In this modified algorithm, numerical computations are made with (A.16) and (A.17) instead of (A.12)-(A.14), the boundedness of the involved variables is then ensured under observability and controllability assumptions. This improvement has been strongly inspired from the new approach presented in Section 3.

FDI algorithm. The application of the GLR test is almost the same as in A.1.

\section{A.3 The revisited Willsky-Jones algorithm applied to the more general fault model}

For the purpose of comparison with the filter proposed in Section 3, we now apply the modified Willsky-Jones algorithm described in A.2 to the fault model (3). First we note that, in the case (4) of a jump-type fault, the model (3) is different from the model (A.1). Because of the additive nature of the fault, the same argument of additive decomposition as in (A.2)-(A.4) holds true. Consequently the state, the one-step ahead state prediction and the output prediction error can be decomposed as the addition of two parts: one solely due to the faultfree assumption and one due to the additive fault. The matrices $\alpha_{k}, \beta_{k}, \rho_{k}$ are thus defined accordingly by:

$$
\begin{aligned}
X_{k} & =X_{k}^{0}+\alpha_{k} \theta \\
\widehat{X}_{k} & =\widehat{X}_{k}^{0}+\beta_{k} \theta \\
\varepsilon_{k} & =\varepsilon_{k}^{0}+\rho_{k} \theta
\end{aligned}
$$

Based on (3), (6) and (10), those matrices $\alpha, \beta, \rho$ defined in (A.18)-(A.20) satisfy for all $\theta$ :

$$
\begin{aligned}
\alpha_{k+1} \theta & =F_{k} \alpha_{k} \theta+\Psi_{k} \theta \\
\beta_{k+1} \theta & =F_{k} \beta_{k} \theta+F_{k} K_{k} \rho_{k} \theta \\
\rho_{k} \theta & =H_{k}\left(\alpha_{k}-\beta_{k}\right) \theta
\end{aligned}
$$

from which, defining

$$
\gamma_{k} \triangleq \alpha_{k}-\beta_{k}
$$

and noting that $\alpha_{k}, \beta_{k}, \rho_{k}$ and thus $\gamma_{k}$ do not depend on $\theta$ as we know from Section 3, the following recursive formula is obtained:

$$
\gamma_{k+1}=F_{k}\left(\mathbf{I}-K_{k} H_{k}\right) \gamma_{k}+\Psi_{k}=\Gamma_{k+1}
$$


With (A.23)-(A.25), we get that (A.20) coincides with (11), namely the computations in Section 3 provide the same signature of the fault on the innovation as here.

\section{Acknowledgements}

The authors greatfully acknowledge the help from Paulo Lopes dos Santos and his co-authors of [37] who provided them with data and information about the example studied in Subsection 6.2. The authors also acknowledge useful comments by the Reviewers and the Associate Editor that helped improving an earlier version of the paper.

\section{References}

[1] M. Basseville. Information criteria for residual generation and fault detection and isolation. Automatica, 33(6):783-803, 1997.

[2] M. Basseville and A. Benveniste, editors. Detection of Abrupt Changes in Signals and Dynamical Systems, volume 77 of LNCIS. Springer, Berlin, 1985.

[3] M. Basseville and I. Nikiforov. Detection of Abrupt Changes - Theory and Application. Prentice Hall, Englewood Cliffs, 1993.

[4] M. Basseville and I.V. Nikiforov. Fault isolation for diagnosis: nuisance rejection and multiple hypotheses testing. Annual Reviews in Control, 26(2):189202, 2002.

[5] D. Berdjag, C. Christophe, V. Cocquempot, and B. Jiang. Nonlinear model decomposition for robust fault detection and isolation using algebraic tools. Int. Jal Innovative Computing, Information and Control, 2(6):1337-1354, 2006.

[6] M. Blanke, M. Kinnaert, J. Lunze, J. Schröder, and M. Staroswiecki. Diagnosis and Fault-Tolerant Control (2nd ed.). Springer, Berlin, 2006.

[7] J. Bokor and G. Balas. Detection filter design for LPV systems - A geometric approach. Automatica, 40(3):511-518, 2004.

[8] J. Bokor and Z. Szabó. Fault detection and isolation in nonlinear systems. Annual Reviews in Control, 33(2):113-123, 2009.

[9] A. Casavola, D. Famularo, G. Franzè, and M. Sorbara. A fault-detection, filter-design method for linear parameter-varying systems. Proc. Institution of Mechanical Engineers, Part I: Jal Systems and Control Engineering, 221(6):865-873, 2007.

[10] A. Casavola and G. Gagliardi. Fault detection and isolation of electrical induction motors via LPV fault observers. In Proc. 8th IFAC Safeprocess, pages 800-805, Mexico, August 2012.

[11] J. Chen and R.J. Patton. Optimal filtering and robust fault diagnosis of stochastic systems with unknown disturbances. IEE Proc. Control Theory and Applications, 143(1):31-36, 1996.

[12] J. Chen and R.J. Patton. Robust Model-Based Fault Diagnosis for Dynamic Systems. Kluwer, Boston, 1999.

[13] R. Chen, D.L. Mingori, and J.L. Speyer. Opti- mal stochastic fault detection filter. Automatica, 39(3):377-390, 2003.

[14] R.H. Chen and J.L. Speyer. A generalized leastsquares fault detection filter. Int. Jal Adaptive Control Signal Processing, 14(7):747-757, 2000.

[15] W.H. Chung and J.L. Speyer. A game theoretic fault detection filter. IEEE Trans. Automatic Control, 43(2):143-161, 1998.

[16] S. De Lira, V. Puig, and J. Quevedo. Fault detection and isolation of a real PEM fuel cell using interval LPV observers. In Proc. 8th IFAC Safeprocess, pages 90-95, Mexico, August 2012.

[17] C. De Persis and A. Isidori. A geometric approach to nonlinear fault detection and isolation. IEEE Trans. Automatic Control, 46(6):853-865, 2001.

[18] S.X. Ding. Model-based Fault Diagnosis Techniques: Design Schemes, Algorithms, and Tools. Springer, Berlin, 2008.

[19] A. Edelmayer, J. Bokor, F. Szigeti, and L. Keviczky. Robust detection filter design in the presence of time-varying system perturbations. Automatica, 33:471-475, 1997.

[20] M. Fliess, C. Join, and H. Sira-Ramirez. Robust residual generation for linear fault diagnosis: an algebraic setting with examples. Int. Jal Control, 77(14):1223-1242, 2004.

[21] P.M. Frank. Fault diagnosis in dynamic systems using analytical and knowledge based redundancy - A survey and some new results. Automatica, 26(3):459-474, 1990.

[22] P.M. Frank and J. Wunnenberg. Robust fault diagnosis using unknown input observer schemes. In [?], pages 47-98. Prentice-Hall Int., 1989.

[23] J.J. Gertler. Fault Detection and Diagnosis in Engineering Systems. Marcel Dekker, New York, 1998.

[24] F. Gustafsson. Adaptive Filtering and Change Detection. Wiley, New York, 2000.

[25] D. Henry. Structured fault detection filters for LPV systems modeled in an LFR manner. Int. Jal Adaptive Control Signal Processing, 26(3):190-207, 2012.

[26] D. Henry, A. Zolghadri, J. Cieslak, and D. Efimov. A LPV approach for early fault detection in aircraft control surfaces servo-loops. In Proc. 8th IFAC Safeprocess, pages 806-811, Mexico, August 2012.

[27] M. Hou and P.C. Muller. Disturbance decoupled observer design: a unified viewpoint. IEEE Trans. Automatic Control, 39(6):1338-1341, 1994.

[28] M. Hou and R.J. Patton. Optimal filtering for systems with unknown inputs. IEEE Trans. Automatic Control, 43(3):445-449, 1998.

[29] I. Hwang, S. Kim, Y. Kim, and C.E. Seah. A survey of fault detection, isolation, and reconfiguration methods. IEEE Trans. Control Systems Technology, 18(3):636-653, 2010.

[30] R. Isermann. Supervision, fault-detection and faultdiagnosis methods - An introduction. Control Engineering Practice, 5(5):639-652, 1997.

[31] R. Isermann. Fault Diagnosis Systems: An Introduction From Fault Detection To Fault Tolerance. 
Springer, Berlin, 2005.

[32] A.H. Jazwinski. Stochastic Processes and Filtering Theory. Academic Press, New York, 1970.

[33] Z. Ji, R. Zhu, and F. Ding. On the fault diagnosis of linear time-varying systems. In Proc. IEEE Int. Conf. on Mechatronics and Automation (ICMA'05), volume 2, pages 811-814, Niagara Falls, Ontario, July 2005.

[34] L. Keviczky, J. Bokor, F. Szigeti, and A. Edelmayer. Modeling time varying system perturbations: Application to robust change detection and identification. In Proc. 12th IFAC World Congress, volume 7, pages 517-520, Sydney, July 1993.

[35] X. Li, S.-F. Mo, and K. Zhou. Fault detection for linear discrete time-varying systems. In Proc. 49 th IEEE Conf. on Decision and Control, pages 762767, Atlanta, December 2010.

[36] X. Li and K. Zhou. A time domain approach to robust fault detection of linear time-varying systems. Automatica, 45(1):94-102, 2009.

[37] P. Lopes dos Santos, T.-P. Azevedo-Perdicoúlis, J.A. Ramos, J.L. Martins de Carvalho, G. Jank, and J. Milhinhos. An LPV modeling and identification approach to leakage detection in high pressure natural gas transportation networks. IEEE Trans. Control Systems Technology, 19(1):77-92, 2011.

[38] M.-A. Massoumnia. A geometric approach to the synthesis of failure detection filters. IEEE Trans. Automatic Control, 31(9):839-846, 1986.

[39] P.L. Negre, V. Puig, and I. Pineda. Interval LPV identification and fault diagnosis of a real wind turbine. In Proc. 16th Symp. on System Identification (SYSID'12), pages 1689-1694, Brussels, July 2012. IFAC.

[40] R.J. Patton, P.M. Frank, and R. Clarke, editors. Issues of Fault Diagnosis for Dynamic Systems. Springer, London, 2000.

[41] P. Rosa, C. Silvestre, J.S. Shamma, and M. Athans. Fault detection and isolation of LTV systems using set-valued observers. In Proc. 49th IEEE Conf. on Decision and Control, pages 768-773, Atlanta, December 2010.

[42] P.A.N. Rosa, P. Casau, C. Silvestre, S.M. Tabatabaeipour, and J. Stoustrup. A set-valued approach to FDI and FTC: Theory and implementation issues. In Proc. 8th IFAC Safeprocess, pages 1281-1286, Mexico, August 2012.

[43] A. Ticlea and G. Besançon. Adaptive observer for discrete time state affine systems. In Proc. 16th Symp. on System Identification (SYSID'12), pages 1245-1250, Brussels, July 2012. IFAC.

[44] R. Tóth, J. Willems, P. Heuberger, and P. Van den Hof. The behavioral approach to linear parametervarying systems. IEEE Trans. Automatic Control, 56(11):2499-2514, 2011.

[45] A. Varga. On parametric solution of fault detection problems. In Proc. 18th IFAC World Congress, pages 6697-6702, Milan, August 2011. IFAC.

[46] A. Varga, S. Hecker, and D. Ossmann. Diagnosis of actuator faults using LPV-gain scheduling techniques. In Proc. Guidance Navigation and Control Conf. (GNC'11), Portland, August 2011. AIAA.

[47] A. Varga and D. Ossmann. LPV-model based identification approach of oscillatory failure cases. In Proc. 8th IFAC Safeprocess, pages 1347-1352, Mexico, August 2012.

[48] N. Viswanadham, J.H. Taylor, and E.C. Luce. A frequency-domain approach to failure detection and isolation with application to GE-21 turbine engine control systems. Control Theory and Advanced Technology, 3(1):45-72, 1987.

[49] A.S. Willsky and H. Jones. A generalized likelihood ratio approach to state estimation in linear systems subjects to abrupt changes. In Proc. IEEE Conf. on Decision and Control, volume 13, pages 846-853, Phoenix, November 1974.

[50] A.S. Willsky and H. Jones. A generalized likelihood ratio approach to the detection and estimation of jumps in linear systems. IEEE Trans. Automatic Control, 21(1):108-112, 1976.

[51] A. Xu and Q. Zhang. Residual generation for fault diagnosis in linear time-varying systems. IEEE Trans. Automatic Control, 49(5):767-772, 2004.

[52] Q. Zhang. Adaptive observer for multiple-inputmultiple-output (MIMO) linear time-varying systems. IEEE Trans. Automatic Control, 47(3):525529, 2002.

[53] Q. Zhang. An adaptive observer for sensor fault estimation in linear time varying systems. In Pavel Zítek, editor, Proc. 16th IFAC World Congress, pages 137-142, Prague, 2005.

[54] Q. Zhang. Revisiting different adaptive observers through a unified formulation. In Proc. 44th IEEE Conf. on Decision and Control and European Control Conf., pages 3067-3072, Seville, December 2005.

[55] Q. Zhang. From adaptive observers to decoupled state and parameter estimations. In Gang Tao and Jing Sun, editors, Advances in Control Systems Theory and Applications, pages 242-255. USTC Press, Hefei, CN, 2009.

[56] M. Zhong, S.X. Ding, and E. Ding. Optimal fault detection for linear discrete time-varying systems. Automatica, 46(8):1395-1400, 2010.

[57] M. Zhong, S.X. Ding, Q.-L. Han, and Q. Ding. Parity space-based fault estimation for linear discrete time-varying systems. IEEE Trans. Automatic Control, 55(7):1726-31, 2010.

[58] M. Zhong, D. Zhou, and S.X. Ding. On designing $\mathcal{H}_{\infty}$ fault detection filter for linear discrete timevarying systems. IEEE Trans. Automatic Control, 55(7):1689-1695, 2010. 